\title{
Determination of Source Parameters of Explosions and Earthquakes by Amplitude Equalization of Seismic Surface Waves
}

\section{Underground Nuclear Explosions ${ }^{1}$}

\author{
M. N. Toksöz, A. Ben-Menahem, ANd D. G. Harkrider \\ Seismological Laboratory \\ California Institute of Technology, Pasadena
}

\begin{abstract}
A method of determining the source parameters of explosions and earthquakes from the amplitude spectrums of seismic surface waves is described. The method, called amplitude equalization, involves the correction of the ground displacement spectrum for the propagation effect. This is accomplished by multiplying it numerically with the inverse of the frequency response of the layered medium. The result is the amplitude spectrum of the source function, which may be interpreted by itself or jointly with the initial phase spectrum to determine the source-time variation. The spectrums of the Rayleigh waves from underground nuclear explosions are compared and the source-time function is interpreted using the amplitude equalization method. The time variation of the pressure pulse at the boundary of the elastic zone is found to be of the form $p(t)=P_{0} t e^{-\eta t}$, where $\eta$ is a parameter which depends on the yield of the explosion and on the medium. For the events studied, the breadth of the pulse increased ( $\eta$ decreased) with the yield of the explosion.
\end{abstract}

Introduction. The study of seismic waves generated by underground nuclear explosions helps us to understand the mechanism of seismic events in general. Relatively little is now known about the mechanism of seismic wave generation at the source of explosions and small earthquakes. We have studied by the amplitude equalization method the seismic surface waves radiated from three underground nuclear explosions and one collapse event to determine the source function.

The source functions of seismic waves generated by underground nuclear explosions were investigated from two directions: far-field deductions and near-field measurements. The farfield deductions were calculated from the seismic body and surface waves recorded at distant stations. A comprehensive list of references on this subject is given by Press et al. [1963]. We will mention two studies, however, which deal directly with the particular subject of our interest. Smith [1963] studied the source space and time dependence of seismic waves generated

\footnotetext{
1 Contribution 1262, Division of Geological Sciences, California Institute of Technology, Pasadena.
}

by underground explosions and the associated collapse events using the spectral ratios of short-period ( 0.3 to $5 \mathrm{cps}) S V$ waves and the polarity of $P$ waves. He concluded that the explosion and collapse had explosive spherical and downward single forces, respectively, with the suggestion of a slightly sharper pulse for the explosion relative to the collapse. Brune and Pomeroy [1963] used the phase equalization technique and the relatively long-period (10 to $35 \mathrm{sec})$ Rayleigh waves to determine the space and time dependence of the source. For explosions in alluvium and accompanying collapses, they deduced the space functions to be explosive and implosive, respectively. The time dependence of the sources were explained as being step functions for both events.

The near-source measurements of pressure, acceleration, velocity, and displacements are reported by various investigators [Haskell, 1961; Weart, 1962; Bass et al., 1963; Bishop, 1963; Kisslinger, 1963; Wistor et al., 1963]. Most of the measurements indicate a more complicated time dependence than a step function. Furthermore, the source-time function seems to vary with the medium in which the shot is fired and the yield of the explosion. Un- 
fortunately, there are not enough of these measurements for the larger explosions just outside the nonlinear zone around the explosion to permit us to interpret the source function of the seismic waves accurately.

In this paper, we use the spectrums of the Rayleigh waves in conjunction with the available near-source information to determine the source functions and relative yields of two nuclear explosions and a collapse event. In the first section of the paper the amplitude equalization method is described; in the second and third sections the observed Rayleigh wave data from the events and the determination of the source functions are described.

Amplitude equalization. The method of amplitude equalization for determining source functions has become practical since the amplitude response of multilayered mediums can be obtained [Harkrider, 1964]. The vertical and the radial components of the Rayleigh wave motion and the transverse Love wave motion recorded at the surface of the layered medium, at a point distant from the source, can be expressed in the frequency domain as

$$
\begin{aligned}
& w_{0}(\omega)=C_{1}(2 \pi r)^{-1 / 2} \\
& \cdot \exp \left(-\gamma_{1} r\right) L_{w}(\omega) S_{w}(\omega) T(\omega) I(\omega) \\
& \cdot \exp \left[i\left(\omega t-k_{1} r-\phi_{s}-\phi_{t}-\phi_{\imath}-3 \pi / 4\right)\right] \\
& q_{0}(\omega)=C_{1}(2 \pi r)^{-1 / 2} \\
& \quad \cdot \exp \left(-\gamma_{1} r\right) L_{a}(\omega) S_{q}(\omega) T(\omega) I(\omega) \\
& \quad \cdot \exp \left[i\left(\omega t-k_{1} r-\phi_{s}-\phi_{t}-\phi_{\imath}-\pi / 4\right)\right] \\
& v_{0}(\omega)=C_{2}(2 \pi r)^{-1 / 2} \\
& \quad \cdot \exp \left(-\gamma_{2} r\right) L_{v}(\omega) S_{v}(\omega) T(\omega) I(\omega) \\
& \quad \cdot \exp \left[i\left(\omega t-k_{2} r-\phi_{\star}-\phi_{\iota}-\phi_{\imath}+\pi / 4\right)\right]
\end{aligned}
$$

where $w_{0}, q_{0}$, and $v_{0}$ are the vertical, radial, and transverse components of the motion at the surface at a radial distance $r$ from the epicenter. $C_{1}$ and $C_{2}$ are constants. $L(\omega), S(\omega), T(\omega)$, and $I(\omega)$ are, respectively, the amplitude responses of the layered medium, source space function, source-time function, and amplitude response of the recording seismograph. $\omega$ is the angular frequency and $k$ is the wave number. $\phi_{0}, \phi_{t}$, and $\phi_{t}$ are the phase delays of the source and time functions and that of the seismo- graph. The source function describes the geometry of the source and indicates whether it is an explosive point source, a single force, a couple, or a double couple; the time function describes the variation of force with time such as an impulse or a step.

In equation 1 the layering response $L(\omega)$ and the source function $S(\omega)$ are shown as independent factors. In general, however, $S(\omega)$ is also a function of the layering through its dependence on source depth [Ben-Menahem and Harkrider, 1964]. For example, for a spherical pressure source of radius $a$ which is buried at a depth $z=h(h>a)$, the impulse response is given by [Harkrider, 1964]

$$
\begin{aligned}
& L S_{w}(\omega)=2 a^{3} P_{0} k^{1 / 2} \\
& \cdot \frac{\frac{1}{2 \mu_{m}}\left\lceil\frac{\sigma_{m}(h)}{\dot{w}_{n} / c}\right\rceil-\left\lceil\frac{\dot{u}_{m}(h)}{\dot{w}_{0}}\right\rceil}{\left[\left(1-a^{2} k_{\beta_{m}}{ }^{2} / 4\right)^{2}+k_{\alpha m}{ }^{2} a^{2}\right]^{1 / 2}} A_{R}(\omega) \\
& L S_{a}(\omega)=2 a^{3} P_{0} k^{1 / 2}\left(\frac{\dot{u}_{0}}{\dot{w}_{0}}\right) \\
& \cdot \frac{\frac{1}{2 \mu_{m}}\left[\frac{\sigma_{m}(h)}{\dot{w}_{0} / c}\right]-\left[\frac{\dot{u}_{m}(h)}{\dot{w}_{0}}\right]}{\left[\left(1-a^{2} k_{\beta_{m}}{ }^{2} / 4\right)^{2}+k_{\alpha_{m}}{ }^{2} a^{2}\right]^{1 / 2}} A_{R}(\omega) \\
& L S_{v}(\omega)=0
\end{aligned}
$$

where $P_{0}$ is the pressure, $\dot{u}, \dot{w}$ refer to time derivatives of the radial and vertical components of the displacements, $c$ is the phase velocity, and $\mu$ is the rigidity. Subscripts 0 and $m$ signify the surface and the layer in which the source is located, respectively. $k_{a}=\omega / \alpha$ and $k_{\beta}=\omega / \beta$, where $\alpha$ and $\beta$ are compressional and shear velocities. $A_{R}$ is a function of the layered medium that is independent of the source. The factor that contains the source depth is defined as

$$
\frac{1}{2 \mu_{m}}\left[\frac{\sigma_{m}(h)}{\dot{w}_{0} / c}\right]-\frac{\dot{u}_{m}(h)}{\dot{w}_{0}}=-\frac{1}{2} k_{\beta_{m}}^{2}\left[\frac{\varphi_{m}(h)}{\dot{w}_{0} / c}\right]
$$

where $\varphi_{m}$ is the compressional wave potential. The formulations for other source functions and for Love waves are given by Harkrider [1964], and they are extended to sources of arbitrary orientation by Ben-Menahem and Harkrider [1964] and by Haskell [1964], using a similar matrix formulation.

Once the response of the layered medium is computed for a given structure and a given 
source, the procedure of determining the time dependence of the source function becomes a straightforward task. The Fourier amplitude spectrums of the recorded Rayleigh waves are corrected for the instrument response and the combined source and layering response by pointwise division. The remaining spectrum is the amplitude spectrum of the source-time function.

It is obvious that we cannot determine both the space and the time dependence of the source uniquely from a single observation. However, if there are recordings at a number of stations around the source, the azimuthal variation can be used to determine the space variation of the source, and then the time function, which is independent of the azimuth, can be fixed uniquely. Furthermore, in the presence of both Love and Rayleigh waves, the spectral ratios of the two wave types can be used to determine the space variation of the source.

The method of amplitude equalization is the direct analog of the phase equalization where the phase spectrum of the source function is obtained. It has several practical advantages and some disadvantages, however, compared with the phase equalization method: (1) Given a wave train, the amplitude spectrum can be determined more accurately than the phase spectrum by Fourier analysis. The error in the phases is especially great at frequencies where either the sine or cosine transform is very small. (2) The phase equalization requires absolute timing of the events. Any error in the origin time of the event or time (clock) correction of the recording introduces frequency-dependent phase errors. Furthermore, determining the phase response of the recording instrument from calibration pulses is more susceptible to errors than determining the amplitude response, because it is difficult to determine the onset of the pulse precisely. (3) The amplitude response of the layered medium, in general, varies more slowly than the phase velocities as the structure is changed. Therefore, inadequate knowledge of the structure introduces relatively less error in the amplitude response than in the propagation phase angle. (4) In phase equalization the observed phase velocities can be used without interpreting the structure. Although this is especially true for long-period waves, the average accuracy of the short-period phase velocities is not very great. (5) A limitation of the amplitude equalization results from the lack of precise data on attenuation coefficients at relatively high frequencies at the present time. This limitation becomes more serious for large epicentral distances.

A comparative study of the Rayleigh waves from nuclear explosions. Rayleigh waves from three underground nuclear explosions (Hardhat, Haymaker, and Sedan) and one collapse event (Haymaker) at the Nevada test site were recorded at four stations (Pasadena, California; Jamestown, California; Ruth, Nevada; Albuquerque, New Mexico). Pertinent information about the explosions and the recording stations is given in Tables 1 and 2 and in Figure 1. Each station is equipped with a set of identical high-sensitivity, narrow-band Benioff seismographs. In this study only the vertical components of Rayleigh waves were used.

The recorded Rayleigh waves were digitized at 1-sec intervals. The wave trains from the digital data are plotted in Figure 2. In this figure the amplitude of each event was normalized to unity. In other words, the absolute amplitude relations are not maintained. Some of the wave trains are plotted upside down (dashed lines). The Rayleigh waves illustrated do not show significant dispersion because of the short epicentral distances and the narrow pass-band of the instrument. The Rayleigh wave pulses

TABLE 1. Explosion Data

\begin{tabular}{|c|c|c|c|c|c|c|c|c|c|}
\hline \multirow[b]{2}{*}{ Explosion } & \multirow[b]{2}{*}{ Date } & \multicolumn{3}{|c|}{$\begin{array}{c}\text { Origin Time, } \\
\text { UT }\end{array}$} & \multicolumn{2}{|c|}{ Location } & \multirow{2}{*}{$\begin{array}{l}\text { Shot } \\
\text { Depth, } \\
\text { m }\end{array}$} & \multirow{2}{*}{$\begin{array}{l}\text { Yield, } \\
\text { kilotons }\end{array}$} & \multirow[b]{2}{*}{ Medium } \\
\hline & & $\mathrm{h}$ & $\mathrm{m}$ & $\mathbf{s}$ & Lat., $\overline{\mathrm{N}}$ & Long., W & & & \\
\hline Hardhat & Feb. 15, 1962 & 18 & 00 & 00.1 & $37^{\circ} 13^{\prime} 35^{\prime \prime}$ & $116^{\circ} 03^{\prime} 34^{\prime \prime}$ & 290 & 6 & Granite \\
\hline Haymaker & June 27, 1962 & 18 & 00 & 00.1 & $37^{\circ} 02^{\prime} 30^{\prime \prime}$ & $116^{\circ} 02^{\prime} 07^{\prime \prime}$ & 410 & 56 & Alluvium \\
\hline $\begin{array}{c}\text { Haymaker } \\
\text { collapse }\end{array}$ & June 27,1962 & 18 & 25 & 00 & $37^{\circ} 02^{\prime} 30^{\prime \prime}$ & $116^{\circ} 02^{\prime} 07^{\prime \prime}$ & 410 & & Alluvium \\
\hline Sedan & 6,1962 & 17 & 00 & 00.1 & $37^{\circ} 10^{\prime} 37^{\prime \prime}$ & $116^{\circ} 02^{\prime} 43^{\prime \prime}$ & 200 & 100 & Alluvium \\
\hline
\end{tabular}


TABLE 2. Station Data

\begin{tabular}{lcccc}
\hline \multirow{2}{*}{ Station } & \multicolumn{2}{c}{ Location } & Distance to & Haymaker \\
Lat., N & Long., W & Haymaker, km & Station Azimuth \\
\hline Albuquerque & $34^{\circ} 57^{\prime} 00^{\prime \prime}$ & $106^{\circ} 27^{\prime} 29^{\prime \prime}$ & 893.8 & $102.2^{\circ}$ \\
Jamestown & $37^{\circ} 56^{\prime} 53^{\prime \prime}$ & $120^{\circ} 26^{\prime} 17^{\prime \prime}$ & 402.1 & $285.8^{\circ}$ \\
Pasadena & $34^{\circ} 08^{\prime} 53^{\prime \prime}$ & $118^{\circ} 10^{\prime} 16^{\prime \prime}$ & 374.8 & $211.7^{\circ}$ \\
Ruth & $39^{\circ} 15^{\prime} 00^{\prime \prime}$ & $115^{\circ} 00^{\prime} 00^{\prime \prime}$ & 261.4 & $20.0^{\circ}$ \\
\hline
\end{tabular}

from all the events recorded at each station are, in general, alike. This is especially true of the Haymaker collapse and the Sedan explosion records. The polarity of the Rayleigh waves from the collapse event is reversed relative to the Haymaker and Sedan explosions. This reversal of polarity has been observed for other explosion and collapse pairs [Smith, 1963; Brune and Pomeroy, 1963]. The Hardhat explosion generated Rayleigh waves different from those of the Haymaker collapse or the Sedan explosion in the direction of Pasadena and Ruth. The Rayleigh waves from the Hardhat explosion vary more significantly from the others at all stations.

The significance of the similarities and the differences between the Rayleigh waves from the different events can be expressed quantitatively using correlation functions. We define a 'correlation coefficient'

$$
r_{x y}=\left[R_{x y}\right]_{M}\left[R_{y x}\right]_{M} /\left[R_{x x}(0) R_{y y}(0)\right]
$$

where $\left[R_{x y}\right]_{y}$ and $\left[R_{y x}\right]_{y}$ are the maximum values of the cross-correlation functions between the time series $x$ and $y$, and the $R_{m x}(0)$ and $R_{y y}(0)$ are the maximums of the autocorrelation functions. The 'correlation coefficients' between the Rayleigh waves from the three explosions and that of the Haymaker collapse are shown in Figure 3. The sign of the coefficients is negative except for the waves from the Hardhat explosion recorded at Pasadena and at Ruth. This again indicates that the polarity of Haymaker collapse is reversed relative to the Haymaker and Sedan explosions. The reversal of the polarity of the Hardhat explosion relative to the other explosions at Ruth and Pasadena suggests a radiation pattern for the Hardhat event which is not symmetric.

The values of the 'correlation coefficients' between the collapse and Sedan are very close to unity at all stations. The similarity of Rayleigh waves at all stations, as well as the lack of Love waves from these events, indicates that the sources of both were alike and symmetric. In this study, we have chosen the collapse as a reference and have compared the properties of the other events to that of the collapse. Since all the epicenters are within a few tens of kilometers from each other, such comparative study

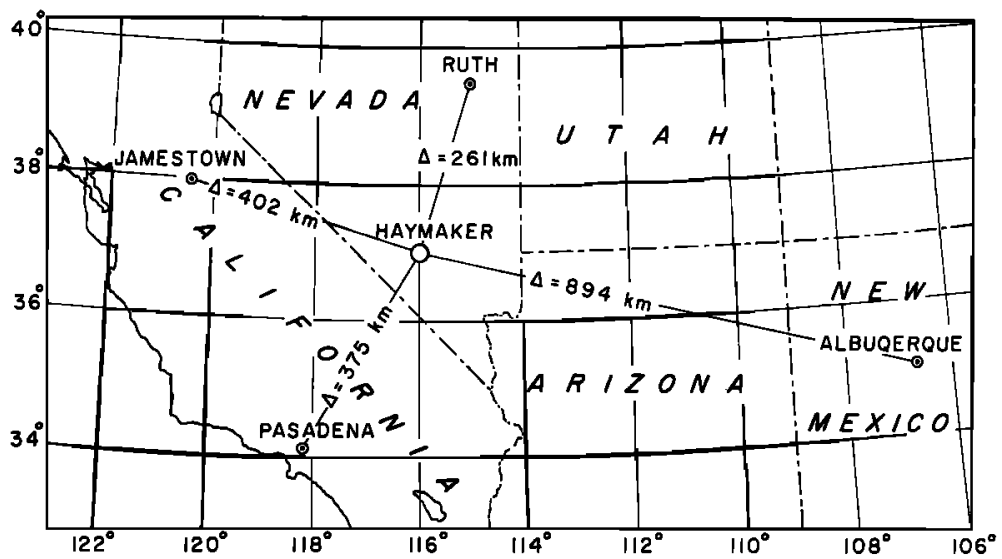

Fig. 1. Map showing the four seismic stations around the Nevada test site. 


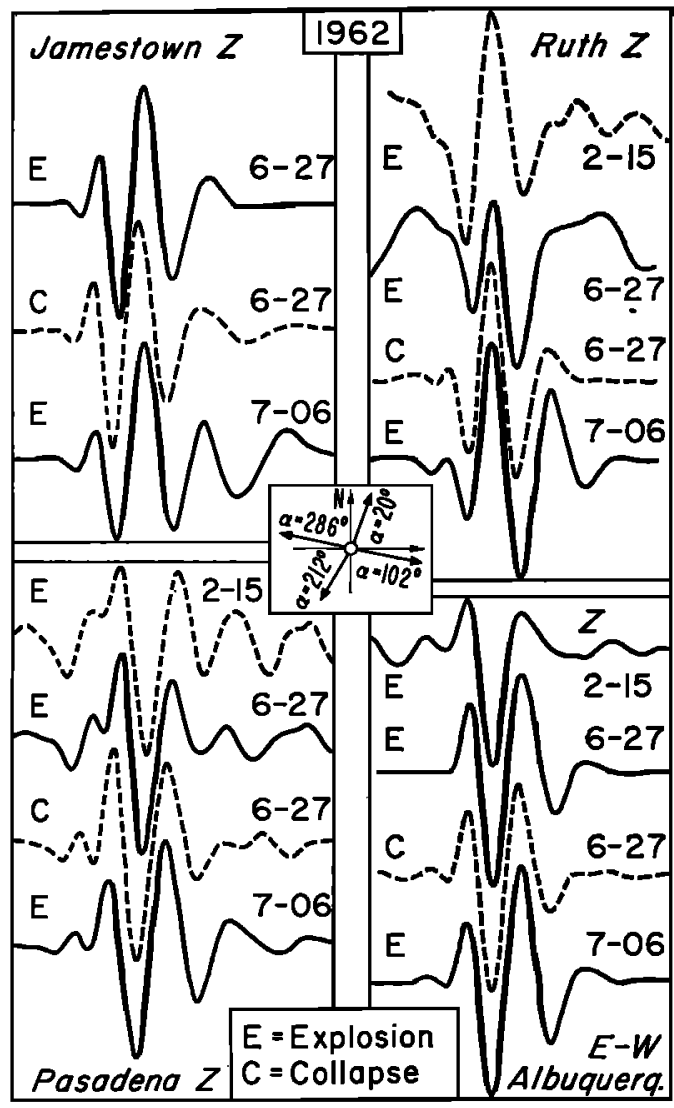

Fig. 2. The Rayleigh wave pulses from three explosions and one collapse event. Maximum amplitudes of all events are equalized to unity. The dashed lines indicate that the polarity was reversed.

indicates the differences between them regardless of the knowledge of the source, medium of propagation, and response of the instruments.

This study is based primarily on the amplitude spectrums. In Figure 4 the Fourier amplitudes of the Rayleigh waves from the Haymaker explosion and the collapse are illustrated. The relative amplitudes are the same as those of the ground motion amplitudes. At Albuquerque and Jamestown the spectrums are almost identical over the entire frequency band. At Pasadena and Ruth the amplitude of the Rayleigh waves from the explosion is about half the amplitude of the collapse. Furthermore, the explosion spectrum is less regular because of interference, which may be caused by local noise or complication in the source radiation. Although four stations are not sufficient to determine the radiation pattern exactly, the complexities presented by the Hardhat and the Haymaker explosions demonstrate a significant regularity. For the Hardhat explosion, the records at Ruth and Pasadena show a reversed polarity. One explanation is that in addition to the symmetric radiation there was a multipolar radiation which was probably due to tectonic strain release. The magnitude of the tectonic release was larger than the Hardhat explosion, and it obscured the radiation pattern of the explosive source. For the Haymaker explosion, where the tectonic strain release was probably smaller than the explosion, partial cancellation occurred in quadrants where the polarities were opposite. A detailed study of the asymmetry of radiation will be described in another paper [Toksöz et al., 1964].

The amplitude spectrums of the explosions were normalized to that of the Haymaker collapse in order to illustrate the differences in the source functions. The spectral ratios shown in Figure 5 represent the ratios in the source functions, since the effects of the propagation and instrument response are cancelled in each case. The ratios of the Haymaker explosion are very close to unity at Jamestown and at Albu-

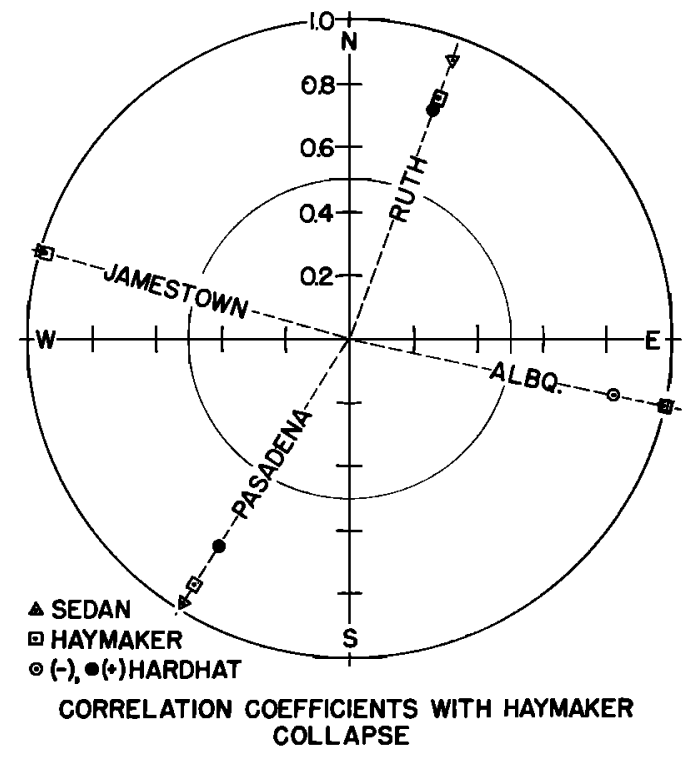

Fig. 3. The 'correlation coefficients' between the Rayleigh waves generated by the explosions and by the collapse. 


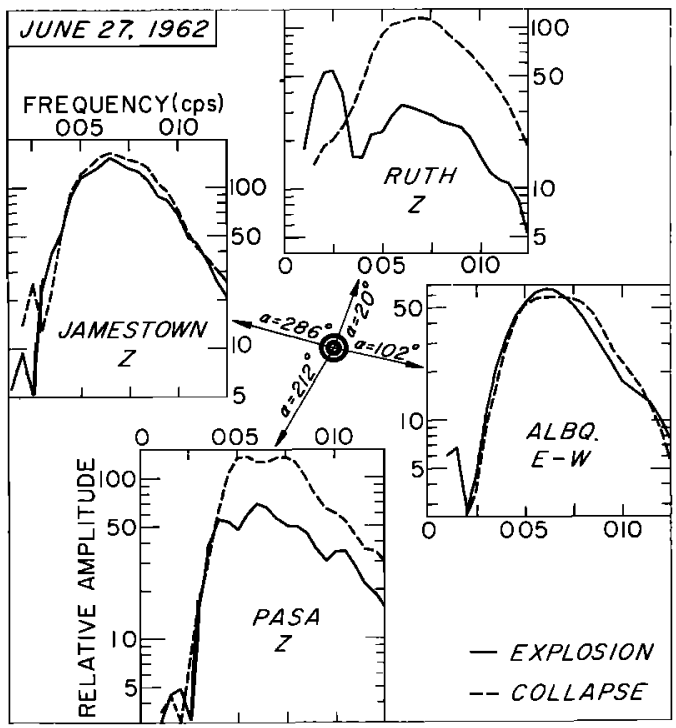

Fig. 4. Amplitude spectrums of Rayleigh waves from the Haymaker explosion and the collapse.

querque, and close to 0.5 at the two other stations. The Hardhat event shows a rapid decrease of relative amplitude with increasing frequency at all four stations. Since we do not know the relative amplitudes of the explosion and the strain release we cannot determine whether the explosion or the strain release is responsible for the behavior of the amplitude spectrum. The spectral ratios of the Sedan explosion show the most regular variation at all stations. This regularity is expected, since both the Sedan explosion and the Haymaker collapse have symmetric radiation. There is one outstanding characteristic of the Sedan spectrums: their relative amplitudes decrease with increasing frequency. In other words, the source of the Sedan explosion had a greater abundance of longer-period Rayleigh waves relative to the Haymaker explosion or collapse events. This indicates that the source function of these events cannot be attributed to a simple explosion or implosion with a time variation independent of yield. If the time variations were independent of yield for these explosions, the amplitude ratios would be constant with frequency. Furthermore, seismic model experiments have shown that the larger explosions generate waves of relatively longer period than the smaller explosions [Gupta and Kisslinger, 1964].

Source function of Rayleigh waves from nuclear explosions. The relative increase of the

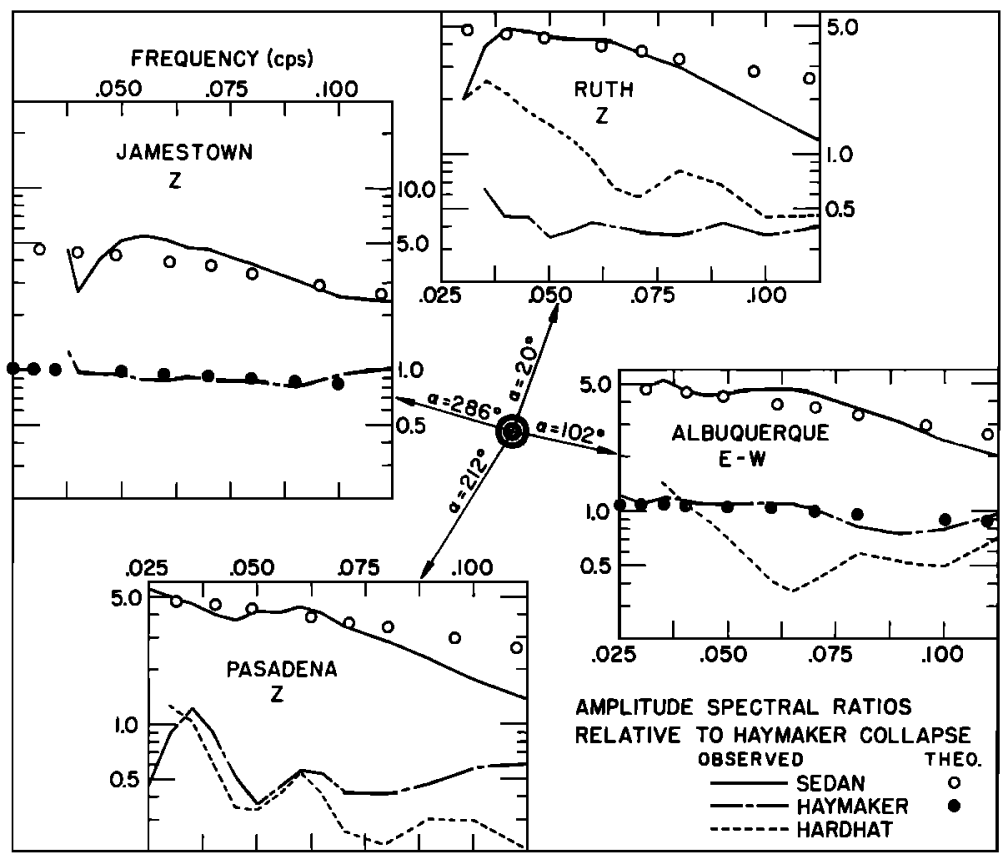

Fig. 5. The ratios of the amplitude spectrums of explosion generated Rayleigh waves to those of collapse. 
long-period components in the spectrums of the Rayleigh waves with increasing yield of the explosion may be due to two factors: the size of the cavity and the variation of time function of the pressure pulse. The relative effect of the size of a spherical source cavity in a half-space depends in a complex way both on the ratio of the cavity radius $a$ to the wavelength $\lambda$ and on the ratio of $\lambda$ to the depth of the cavity $h$. If $a / \lambda \ll 1$ and $a / h<1$, the deviation from a point source is negligible. The second-order approximation based on the theoretical results of Ben-Menaham and Cisternas [1963] yields an approximate finiteness factor of $\left[1-\left(k_{a} a\right)^{2}\right]$, which is applied to the explosive point-source solution for Rayleigh waves in a homogeneous half-space. For a cavity of radius $a=200 \mathrm{~m}$, the perturbation of Rayleigh waves of periods greater than $10 \mathrm{sec}$ is less than $1 \%$. The effects of cavity size applicable for both short- and long-period Rayleigh waves is being investigated in more detail by Cisternas (1964, personal communication). Since any reasonable cavity size fails to explain the strong increase of the long periods in the explosions spectrums with the increasing yields, the effect must be explained in terms of the source functions of the explosions.

A comprehensive study of the generation and the propagation of shock waves around the point of explosion is presented by Zvolinskii [1960] and by Bishop [1963]. Figure 6 illustrates diagrammatically the nonlinear zones around the explosion. From long-range observations we are only able to retrieve the explosion pressure history as it exists at the boundary where the waves become linear. In the nonlinear zone, the pressure pulse (shock wave) decays more rapidly with distance than in the linear zone, since, in addition to the geometric spreading, the shock wave does work on the medium in crushing and heating the material. Also, there is an intrinsic dispersion and resultant pulse distortion in the nonlinear region. These changes depend on the properties of the medium [Duvall, 1962; Bishop, 1963; Kisslinger, 1963]. The broadening of the pulse with increasing distance is common to all mediums. There are other changes, however, which depend on the porosity, phase transitions, compressibility, and viscosity. These changes create instability and result in one or more pressure fronts traveling

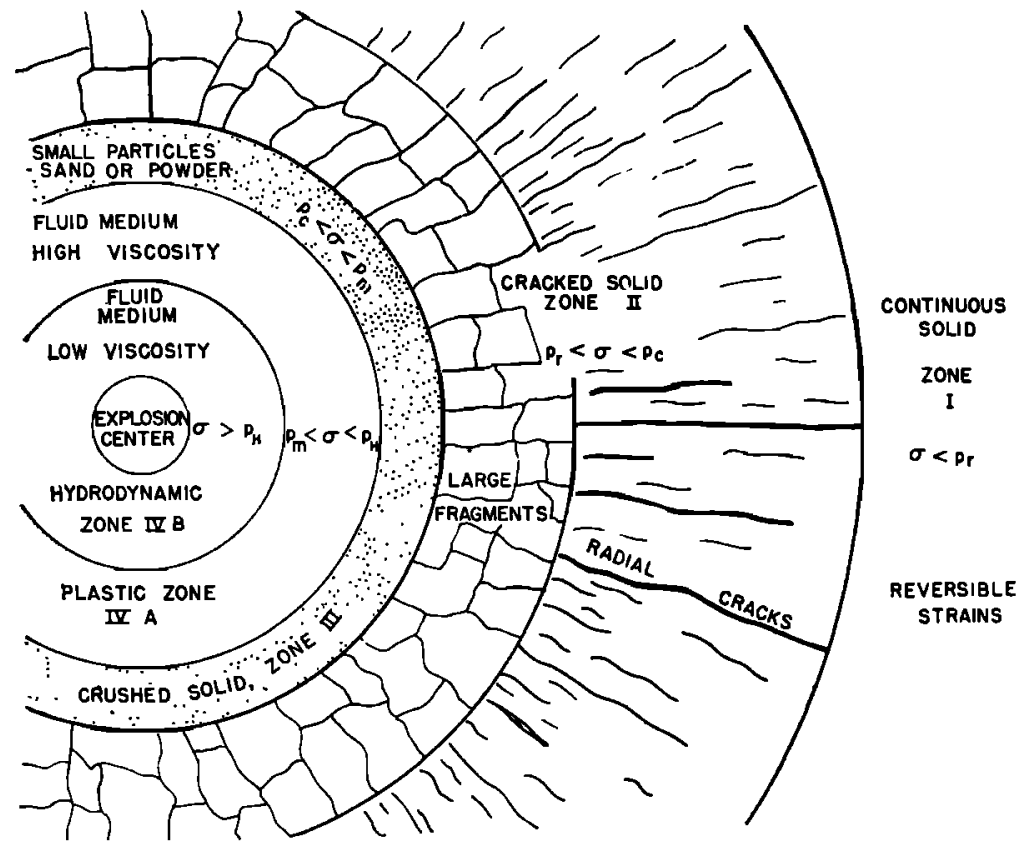

Fig. 6. The schematic diagram of nonlinear zones around an underground explosion [Bishop, 1963]. 
at different velocities. In very general terms, the net effect is either step by step or a continuous rise of pressure to the peak value instead of a single discontinuous jump. In a given medium, the radius of the nonlinear zone increases with the yield. Hence, the pressure pulse from a larger explosion at the boundary of the elastic zone will be broader than that of a smaller explosion.

We can represent the nuclear explosion by an equivalent point source in a medium which behaves linearly everywhere. The time dependence of the equivalent point source is that of the pressure pulse at the boundary of the linear region due to an explosion. On the basis of limited observations [Wistor et al., 1963] and the discussion in the preceding paragraph, we chose to represent the pressure with a function of the form

$$
\begin{array}{ll}
p(t)=P_{0} t^{\xi} e^{-\eta t} & \text { for } \quad t \geq 0 \\
p(t)=0 & \text { for } t<0
\end{array}
$$

where $p(t)$ is the pressure, $t$ is time, $P_{0}$ is a scale factor, $\xi$ and $\eta$ are yield-dependent positive parameters which control the rise time and decay time of the pressure pulse. For example, $\xi=0$ and $\eta=0$ define a step function. The Fourier transform of (5) is

$$
P(\omega)=P_{0} \Gamma(\xi+1)(\eta+i \omega)^{-(\xi+1)}
$$

To simplify the choice of parameters, we fixed the value of $\xi$ using the available pressure measurements. In general, values between $\xi=$ $\frac{1}{3}$ and $\xi=1$ seem to fit the rise time of pressure pulses. Since Sedan and Haymaker explosions are larger than explosions for which near-source measurements are available, we chose $\xi=1$. With this value, the amplitude and the phase spectrums of (6) are

$$
\begin{aligned}
& |P(\omega)|=P_{0} /\left(\eta^{2}+\omega^{2}\right) \\
& \quad \theta(\omega)=-\tan ^{-1}\left[2 \eta \omega /\left(\eta^{2}-\omega^{2}\right)\right]
\end{aligned}
$$

The parameter $\eta$ can be determined from the spectrum of the Rayleigh waves after correcting for the effect of the layered medium and the instrument response. $P_{0}$ is the scale factor related to the yield. It can be estimated by equating the peak pressure at the boundary of the linear region to the limiting strength of the particular medium.
In general, the limiting pressure at which the material ceases to follow the linear stress-strain curve is somewhat less than the maximum reversible pressure. However, where the hydrostatic pressure is much higher than the maximum reversible pressure, the difference between the limiting pressure and the maximum reversible pressure is negligible. Once the information is obtained from the available empirical relations, the yield as well as the radius of the nonlinear zone of a particular explosion can be determined.

The information in Figure 7 is taken from Bishop [1963]. On the same figure, the approximate maximum reversible strength (elastic limit) at 1 bar overpressure is shown. Note that, for all practical purposes, below the reversible pressure the pressure falls off as in the linear zone, as $r^{-1}$. The empirical scaling law that relates the normalization radius to the yield of the explosion is $R_{t}=6.46 W^{1 / 3}$, where $R_{t}$ is

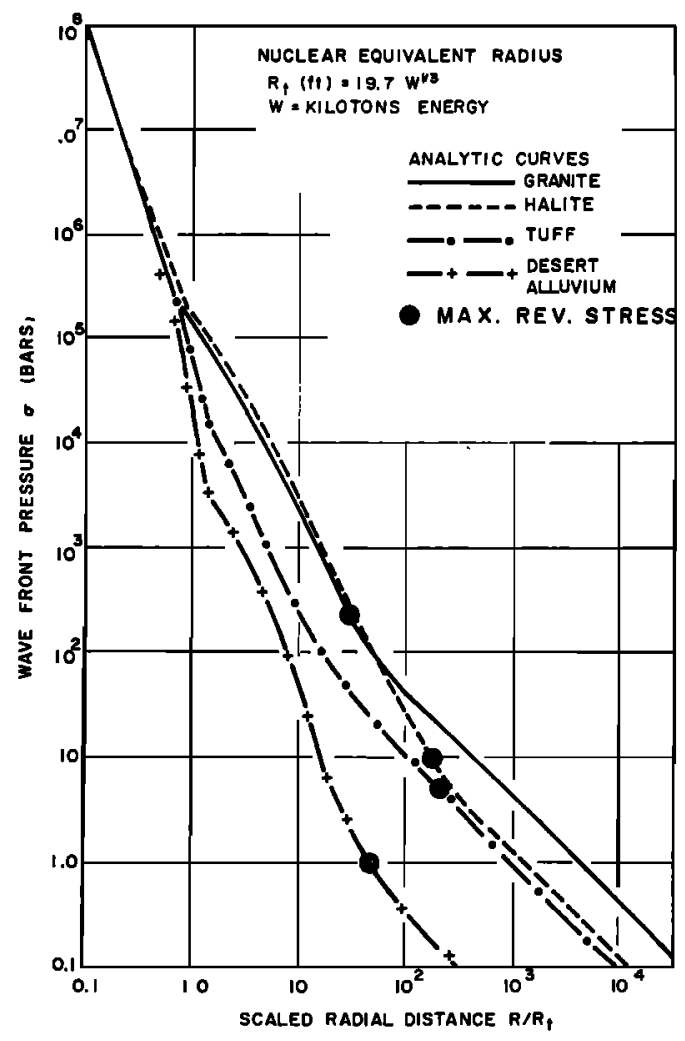

Fig. 7. The pressure fall-off with distance for explosions in various mediums [Bishop, 1963]. 

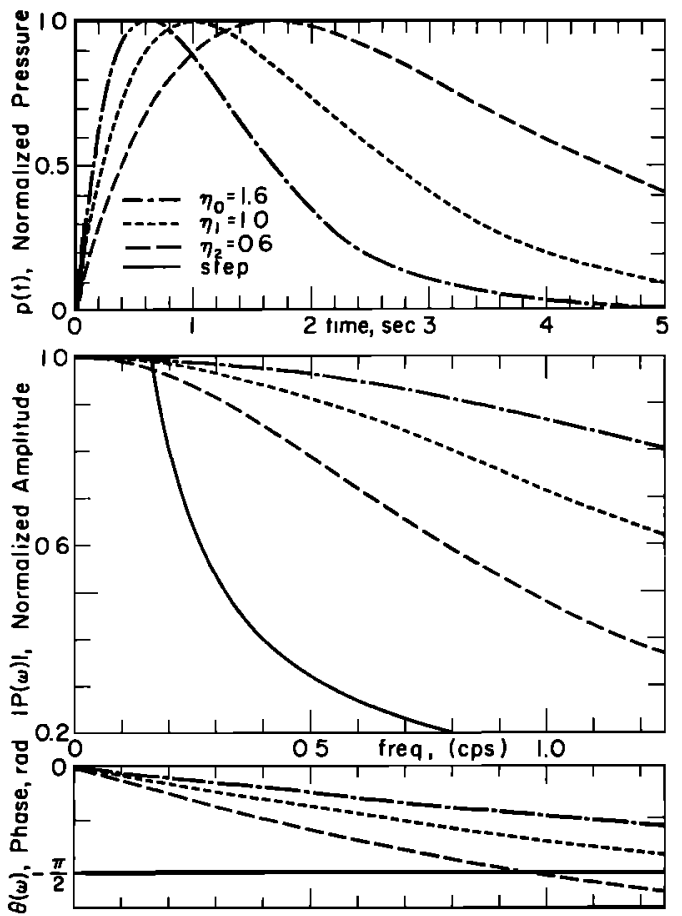

Fig. 8. The source-time functions, amplitude spectrums, and phase spectrums of the Sedan and Haymaker explosions and the Haymaker collapse. The step function is included in the figure for comparison.

in meters, and $W$ is the yield in kilotons (1 kiloton $=4.2 \times 10^{12}$ joules).

From the amplitude ratios, the parameter $\eta$ of the source-time function was determined for the Sedan and Haymaker explosions and the Haymaker collapse. The values are $0.6,1.0$, and $1.6 \mathrm{sec}^{-1}$, respectively. The normalized time functions and their amplitude and phase spectrums are shown in Figure 8, as well as those for a step function. The phase difference between the spectrum of a step function and those proposed in this paper is less than 1.2 radians in the period range 10 to 30 sec. The initial phase measurements, which Brune and Pomeroy [1963] found to be consistent with that of a step function, had an accuracy of about 1.0 radian. Thus, within the limits of experimental error, the decaying source functions determined from the amplitude ratios are consistent with the initial phase data.

The comparison of theoretical amplitude ratios with the observed values for the three events is shown in Figure 5. The general agreement is very good in the more reliable portions of the spectrums ( $f=0.035$ to $f=0.08 \mathrm{cps}$ ). The main emphasis must be placed on the ratio of the Sedan explosion to Haymaker collapse since these were the two most symmetric events and were relatively free of contamination from tectonic strain release.

The next step was to determine whether these models were in agreement with the absolute measurements of the ground displacement. For this purpose the observed amplitude spectrums were corrected for the response of the particular instrument. Instrument responses at each station were determined using the calibration pulses recorded within one week of each event. A typical response curve was shown in a previous publication [Toksöz and Ben-Menahem, 1964]. The ground displacement was then corrected for the layering effect. The response of the layered medium from Nevada test site to Pasadena with an explosive source near the surface and a step-function time variation is shown in Figure 9. The structure used is that given by Alexander [1963]. The spectrums (corrected for instrument, propagation, and explosive nature of the source) were compared with a step-function time dependence and with the time functions shown in Figure 8 . The results (ratio of observed to theoretical) are shown in Figure 10. The correct time function

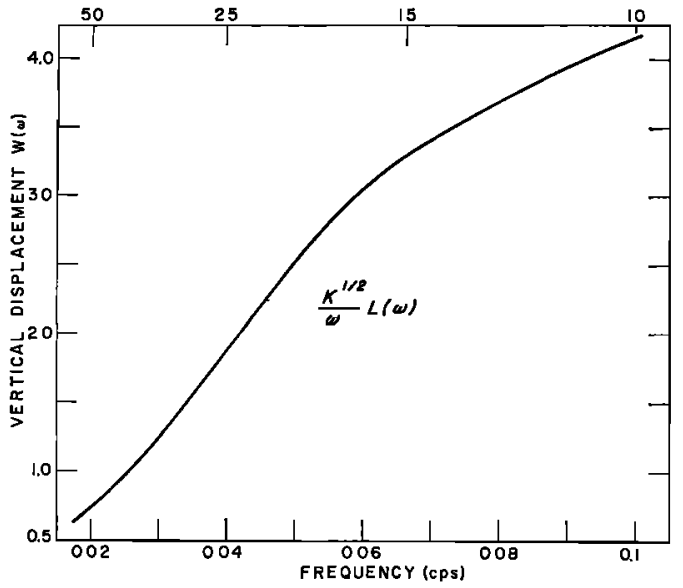

Fig. 9. The response of the layered medium (Nevada test site to Pasadena) to an explosive point source near the surface. The time variation is a step function. 


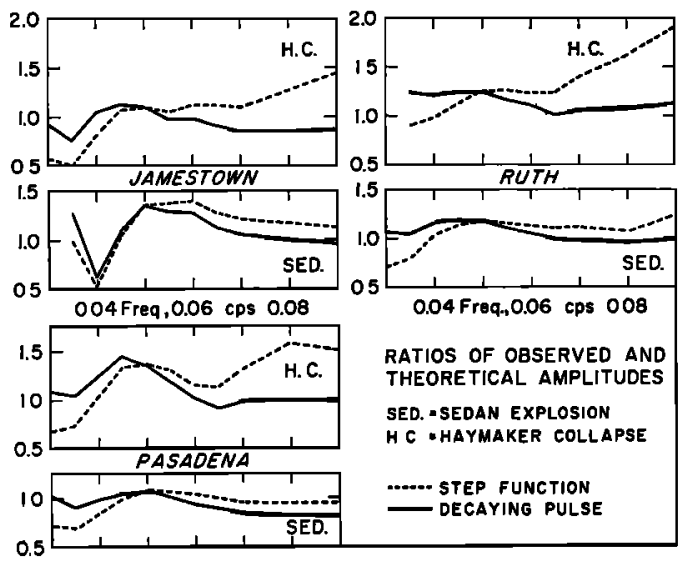

Fig. 10. The ratios of the observed Rayleigh wave amplitude spectrums to those computed theoretically.

must give a constant ratio as a function of frequency. It is seen from Figure 10 that for the collapse the decaying time function gives an almost perfect fit at all stations while the stepfunction ratios increase monotonically with frequency. For the Sedan explosion, the time function decays slowly and the difference between the step and the decaying time function is not as outstanding. Still, however, the decaying time function fits the data better than the step function.

In the above corrections the attenuation was not taken into account. There are no accurate attenuation data for the Rayleigh waves in the period range of our interest. If we assume a constant $Q$ for all frequencies, the attenuation coefficient will increase linearly with frequency and the correction will further enhance the high-frequency components of the source spectrum. This will cause a greater deviation from step-function time dependence for the source. However, the epicentral distances in the present cases are short, and the relative attenuation would not alter the spectrums significantly.

The pressure functions we illustrated are determined from seismic surface waves with periods longer than $10 \mathrm{sec}$. With waves of such long period it is not possible to determine the 'sharp' details of the pressure pulse, since these contribute primarily to the high-frequency end of the spectrums. What we have determined are the smoothed pressure functions equivalent to the actual pressure functions filtered with a band-pass filter with cutoffs at 10 and 40 sec. The details of the pressure fluctuations due to instability of shock waves, and the finite time of transition from the shock wave traveling with subsonic velocity to elastic waves cannot be observed with such a pass-band.

There are two other parameters of the source that can be determined by this method, the radius of the limit of the nonlinear region and the yield of the explosion. The linear region begins where the maximum value of the pressure pulse is equal to the maximum stress in the rock for which a linear stress-strain relation is valid. As mentioned earlier, this limiting stress is very close to the maximum reversible stress at the burial depths of the explosions. Hence we will use the reversible stress in the rest of the paper rather than introducing still another parameter. From (5) the maximum reversible pressure (for $\xi=1$ ) is

$$
P_{\text {rev }}=P_{0} /(\eta e)
$$

Since $\eta$ is determined from the shape of amplitude spectrum, $P_{0}$ can be computed if the reversible stress limit is known. In the linear region the shape of pressure pulse does not change but the amplitude falls off as $r^{-1}$. The radius of the linear boundary is $r_{0}$ for the collapse and $r_{2}$ for the Sedan explosion. The peak pressure of collapse at $r_{2}$ is $\left(r_{0} / r_{2}\right) P_{\text {rev }}$. From the absolute values of the ratio of Sedan and collapse spectrums shown in Figure $5, P_{2} / P_{0}^{\prime}=$ 0.725 where

$$
P_{0}^{\prime}=\left(r_{0} / r_{2}\right) p_{\mathrm{rev}} \eta_{0} e
$$

From these relations, the ratio of the radii of the nonlinear boundaries of Sedan and the collapse is $r_{2} / r_{0}=1.93$. The "cube root scaling law' between the radii and the yields (i.e. $W_{2}^{1 / 3}$ / $\left.W_{0}^{1 / a}=r_{2} / r_{0}\right)$ can be used to compute the ratio of the yield of Sedan explosion to the equivalent yield of collapse, which is $W_{2} / W_{0}=7.2$. In other words, if we take the yield of Sedan to be 100 kilotons, the Haymaker collapse is equivalent to an explosion of 14 kilotons.

The relative radii of nonlinear zones and the relative yields of two explosions fired in different mediums can also be determined by this method. However, the ratio of the maximum reversible stresses is needed in this case.

If the absolute values of the maximum reversible stress and the yield are known, the 
radius of the nonlinear zone can be determined from Figure 7. Taking the maximum reversible pressure to be 10 bars in alluvium at the shot depth and the yield to be 100 kilotons, we find the radius of the nonlinear zone for the Sedan explosion to be about $r_{2}=500 \mathrm{~m}$. Although there is no way of checking the accuracy of this figure, the radius must be larger than the depth of $202 \mathrm{~m}$, since cratering occurred in this case.

Conclusions. 1. The amplitude ratios of the explosion-generated Rayleigh waves recorded at a given station are not, in general, constant with frequency. In some cases (Hardhat, for example), the spectrums are contaminated and the radiation pattern is complicated. This complication may be due to strain release. Where the radiation is symmetric, the spectrums of larger explosions have more longperiod components than short ones.

2. The enhancement of the longer periods with increasing yield of the explosion cannot be explained by an increase in cavity size. This effect is negligible for Rayleigh waves with periods longer than $10 \mathrm{sec}$.

3. The time function of the explosive source, defined as the pressure pulse at the boundary of linear zone, is broader for larger explosions. This broadening is due to the intrinsic dispersion and the distortion of the shock wave as it propagates in the nonlinear zone.

4. The relative yields of explosions and the radii of nonlinear regions around the sources can be determined from the amplitude spectrums of Rayleigh waves using some empirical relations and pressure decay curves. The accuracy of these determinations is limited by the accuracy of the assumptions made in computing the pressure decay curves and the strengths of the explosion mediums.

5. The amplitude equalization method applied in this study and the phase equalization are not merely alternative methods. They complement each other and can be used jointly to determine both the amplitude and the phase spectrums of the source function. From these spectrums the source-time function can be determined uniquely by Fourier synthesis, regardless of its complexity. Neither method, by itself, can achieve a unique determination of the source function.

Acknowledgments. This research was supported by contract AF-49(638)-1337 of the Air
Force Office of Scientific Research as part of the Advanced Research Projects Agency project Vela.

We wish to thank Dr. R. H. Bishop and the Sandia Corporation for permitting us to include two of their figures (Figures 6 and 7 ) in the paper.

\section{ReFERENCES}

Alexander, S. S., Surface wave propagation in the western United States, Ph.D. thesis, California Institute of Technology, Pasadena, 1963.

Bass, R. C., H. L. Hawk, and A. J. Chabai, Dynamic response of geologic solids to large amplitude stress waves, close-in phenomena of buried explosions, Final Rept., SC-4907(RR), pp. 5782, Sandia Corporation, 1963.

Ben-Menahem, A., and A. Cisternas, The dynamic response of an elastic half-space to an explosion in a spherical cavity, J. Math. Phys., 4Q, 112$125,1963$.

Ben-Menahem, A., and D. G. Harkrider, Radiation patterns of surface waves from buried dipolar point sources in a flat stratified earth, J. Geophys. Res., 69, 2605-2620, 1964.

Bishop, R. H., Spherical shock waves from underground explosions, close-in phenomena of buried explosions, Final Rept., $S C-490 \%(R R)$, pp. 115158, Sandia Corporation, 1963.

Brune, J. N., and P. W. Pomeroy, Surface wave radiation patterns for underground nuclear explosions and small-magnitude earthquakes, $J$. Geophys. Res., 68, 5005-5028, 1963.

Duvall, G. E., Concepts of shock wave propagation, Bull. Seismol. Soc. Am., 52, 869-893, 1962.

Gupta, I. N., and C. Kisslinger, Model study of explosion-generated Rayleigh waves in a half space, Bull. Seismol. Soc. Am., 54, 475-484, 1964.

Harkrider, D. G., Surface waves in multilayered elastic media, 1, Rayleigh and Love waves from buried sources in a multilayered elastic halfspace, Bull. Seismol. Soc. Am., 54, 627-680, 1964. Haskell, N. A., A static theory of the seismic coupling of a contained underground explosion, J. Geophys. Res., 66, 2937-2944, 1961.

Haskell, N. A., Radiation pattern of surface waves from point sources in a multi-layered medium, Bull. Seismol. Soc. Am., 54, 377-394, 1964.

Kisslinger, C., The generation of the primary seismic signal by a contained explosion, VESIAC State-of-the-Art-Rept., 85 pp., Acoustics and Seismics Laboratory, Institute of Science and Technology, University of Michigan, 1963.

Press, F., G. Dewart, and R. Gilman, A study of diagnostic techniques for identifying earthquakes, J. Geophys. Res., 68, 2909-2928, 1963.

Smith, S. W., Generation of seismic waves by underground explosions and the collapse of cavities, J. Geophys. Res., 68, 1477-1483, 1963.

Toksöz, M. N., and A. Ben-Menahem, Excitation of seismic surface waves by atmospheric nuclear explosions, J. Geophys. Res., 69, 1639-1648, 1964.

Toksöz, M. N., D. G. Harkrider, and A. BenMenahem, Determination of source parameters by amplitude equalization of seismic surface 
waves, 2, Release of tectonic strain by underground nuclear explosions, in preparation, 1964.

Weart, W. D., Particle motion near a nuclear detonation in halite, Bull. Seismol. Soc. Am., 52, 981-1006, 1962.

Wistor, J. W., J. A. Beyeler, and G. J. Hansen, Long-period displacement gages, close-in phenomena of buried explosions, Final Rept., $S C$ -
4907 $(R R)$, pp. 57-82, Sandia Corporation, 1963.

Zvolinskii, N. V., On the emission of an elastic wave from a spherical explosion in the ground, Soviet J. Appl. Math. and Mech., 24, 166-176, 1960.

(Manuscript received May 25, 1964; revised July 6,1964 .) 